\title{
Therapeutic Anticoagulation May Be Associated with Reduced 14-day Mortality in Mechanically Ventilated Patients with COVID-19
}

\author{
Randi Jenkins, PharmD ${ }^{1}$; Sahil Sheth, PharmD, BCCCP ${ }^{1}$; Breanne Nestor, PharmD, BCCCP ; Adrien Mazer, MD \\ ${ }^{1}$ Department of Pharmacy Services, Anne Arundel Medical Center, Annapolis, Maryland \\ ${ }^{2}$ Department of Critical Care Medicine, Anne Arundel Medical Center, Annapolis, Maryland
}

\begin{abstract}
Purpose: To assess the impact of therapeutic dose versus prophylactic dose anticoagulation regimens on outcomes in mechanically ventilated patients with COVID-19.

Methods: We performed a retrospective cohort analysis of consecutive mechanically ventilated adult patients with COVID-19 admitted to the intensive care unit (ICU) and initiated on anticoagulation from February $1^{\text {st }}$ to May $31^{\text {st }}$, 2020. The primary endpoint was 14 -day mortality. Secondary endpoints included 30-day mortality, hospital length of stay (LOS), duration of mechanical ventilation, major bleeding, and new thromboembolic event.

Results: Of the 121 mechanically ventilated patients with COVID-19, 33 in the therapeutic-dose group and 34 patients in the prophylactic-dose group were included in the final analysis. The therapeutic-dose group had a decreased 14-day mortality compared to the prophylaxis dose group (9.1\% vs $41.2 \%, p=0.004)$. In addition, 30-day mortality was also lower in the therapeutic anticoagulation group (24.2\% vs. 52.9\%, $p=0.024)$. A longer hospital LOS (45.7vs 26 days, $p=0.003$ ) and duration of mechanical ventilation (33.9 vs 13.3 days, $p<0.001)$ were observed in patients on therapeutic anticoagulation in comparison to the prophylaxis dosing group. A higher rate of major bleeding was observed in patients who received therapeutic anticoagulation.

Conclusion: In this analysis of mechanically ventilated COVID-19 patients in the ICU, therapeutic dose anticoagulation was associated with a significantly lower 14-day mortality, but increased bleeding.
\end{abstract}

Keywords: anticoagulation, COVID-19, critical care, mechanical ventilation, venous thromboembolism

\section{Introduction}

SARS-CoV-2 is a respiratory infectious disease that has caused a global pandemic with high rates of morbidity and mortality ${ }^{1}$. Severe presentation of the disease is characterized by a wide array of clinical manifestations such as acute respiratory distress syndrome (ARDS), acute kidney injury, neurologic sequalae and coagulopathy characterized by elevated levels of fibrinogen and D-dimer ${ }^{2}$. Specifically, increased levels of prothrombotic coagulation parameters have been associated with more severe disease and an increased mortality in hospitalized patients ${ }^{3}$. Microthrombi discovered during autopsies of COVID19 patients are hypothesized to play a large role in the pathogenesis of the disease ${ }^{2,4}$. Observational studies have found a thromboembolic incidence of up to $31 \%$ among patients in the ICU admitted with COVID-195. Reported mortality rates have ranged from $36 \%$ to $88 \%$ in those requiring intubation $^{6,7}$. Consequently, institutions established several prophylactic and therapeutic anticoagulation dosing protocols/recommendations in an effort to reduce thromboembolic events and associated morbidity and mortality ${ }^{8}$.

Corresponding author: Sahil Sheth, PharmD, BCCCP

Department of Pharmacy Services

Anne Arundel Medical Center

Annapolis, Maryland

Email: ssheth@aahs.org
Current guidance and recommendations published by organizations such as National Institutes of Health (NIH), World Health Organization (WHO) and International Society on Thrombosis and Hemostasis (ISTH) recommend either prophylactic or intermediate-dose prophylactic anticoagulation in critically ill patients with COVID-19 9,10,11. However, observational reports suggest using therapeutic anticoagulation in high risk patients or those admitted with elevated D-dimers ${ }^{12,13}$. A recent study demonstrated improvements in 21-day survival of mechanically ventilated patients who received therapeutics doses of anticoagulation ${ }^{13}$. In this study, we assessed the impact of therapeutic dose versus prophylactic dose anticoagulation on 14-day mortality in mechanically ventilated patients with COVID-19.

\section{Methods \\ Patients. This was a retrospective single-center study conducted at a 389-bed community teaching hospital in Maryland. The institution has 28 ICU beds and utilizes EPIC electronic medical record (EMR) system. All mechanically ventilated adult patients with COVID-19 admitted to the ICU who received unfractionated heparin or low molecular weight heparin between February $1^{\text {st }} 2020$ and May $31^{\text {st }} 2020$ were evaluated for inclusion. COVID-19 positivity was defined as a laboratory confirmed SARS-CoV-2 infection as determined by polymerase chain reaction (PCR) or other commercial or public health assay in any specimen. Patients on less than 72 hours of mechanical ventilation, receiving anticoagulation prior to admission or transferred from (or to) another acute care facility}


outside of the health system were excluded from the study. Additional exclusion criteria included patients who were pregnant or in the peripartum period and those receiving anticoagulation for indications other than COVID-19. The institutional Clinical Research Committee reviewed this study protocol (IRBNet ID 1618531-1) in June 2020 and deemed it to be IRB exempt.

Data Collection. Demographic and baseline data collected using EMR included patient age, sex, body mass index (BMI), Acute Physiology and Chronic Health Evaluation (APACHE) II Score, Charlson Comorbidity Index, D-dimer, $\mathrm{PaO} 2 / \mathrm{FiO} 2$ ratio, fibrinogen, C-reactive protein (CRP) and ferritin. All baseline demographic and laboratory data was recorded upon ICU admission. Clinical data collected included anticoagulant type, anticoagulant dosing regimen, 14-day mortality, 30-day mortality, hospital LOS, duration of mechanical ventilation, major bleeding and new thromboembolic event. Other data collection points included rate of crossover between groups, protocol compliance and receipt of other COVID-19 therapies. Anticoagulation dosing regimen was initiated and/or continued at the discretion of the attending provider in addition to a health system guidance developed by Department of Pharmacy Services in collaboration with medical leadership. The protocol was approved by the Health System COVID-19 Clinical Management Workgroup and Pharmacy and Therapeutics Committee (Figure 1).

Study Groups. Prophylactic anticoagulation group consisted of patients who received either standard or intermediate dose prophylaxis with unfractionated heparin (UFH) or enoxaparin. Standard dose thromboprophylaxis was defined as UFH dosed at 5000 units subcutaneously every 8 to 12 hours or enoxaparin dosed at 40 or 30 milligram $(\mathrm{mg})$ subcutaneously daily per patient's renal function. Intermediate dose thromboprophylaxis was defined as patients receiving unfractionated heparin dosed at 7500 units every 8 hours or enoxaparin dosed at $40 \mathrm{mg}$ every 12 hours per patient's renal function.

Therapeutic anticoagulation group consisted of patients who received an intravenous (IV) UFH infusion with target activated partial thromboplastin time (aPTT) of 55 to 77 seconds or enoxaparin dosed at $1 \mathrm{mg}$ per kilogram $(\mathrm{kg})$ every 12 or 24 hours per patient's renal function. In addition to the type and dose of anticoagulant, patients must have been initiated on the regimen within the first 72 hours of mechanical ventilation and then continued for at least 72 hours to be classified in therapeutic or prophylaxis dosing groups.

A new thromboembolic event was defined as suspected by the attending provider (without an alternative diagnosis) or confirmed via ultrasonography, computed tomography or other imaging tests. Major bleeding was defined per WHO grade 3 or 4 such as bleeding requiring red blood cell transfusion over routine transfusion needs, bleeding associated with moderate or major hemodynamic instability, fatal bleeding or a bleed involving the central nervous system on imaging study with or without dysfunction. Crossover was defined as any therapeutic anticoagulation regimen which was switched to prophylactic regimen or vice versa not due to bleeding diathesis or thromboembolism.

Study Outcomes. The primary endpoint measured was 14-day mortality. Secondary endpoints included 30-day mortality, duration of mechanical ventilation, major bleeding, and new thromboembolic event. Other pre-defined endpoints included hospital LOS.

Statistical Analysis. For comparison of categorical variables between the two groups, Fisher exact test or chi-squared test were used as appropriate. Fisher's test was used to compare 14-day mortality, 30-day mortality, new thromboembolic event, and major bleed between the two groups. Wilcoxon sum test was used for comparison of continuous variables such as hospital LOS. $P$ values less than 0.05 were deemed to be significant.

\section{Results}

A total of 121 mechanically ventilated adult patients with COVID-19 admitted to the ICU who received heparin or enoxaparin during the study period were identified. A total of 33 patients in the therapeutic dose group and 34 patients in the prophylaxis dose group were included in the final analysis (Figure 2). There were no statistical differences between patient age, sex, $\mathrm{BMI}, \mathrm{PaO} 2 / \mathrm{FiO}_{2}$ ratio on intubation, ferritin level, APACHE II score, and Charlson Comorbidity Index between the groups. Patients in therapeutic dose group had significantly higher $\mathrm{d}$-dimer, fibrinogen, and CRP levels (Table 1).

The therapeutic anticoagulation group had a statistically significant decrease in 14-day mortality compared to the prophylaxis dose group (9.1\% vs $41.2 \%, p=0.003)$. 30-day mortality was also lower in the therapeutic anticoagulation group $(24.2 \%$ vs $52.9 \%, p=0.016)$. A longer duration of mechanical ventilation ( 39.9 vs 13.3 days, $p<0.001$ ) and hospital LOS (45.7 vs 26 days, $p=0.003$ ) was observed in patients on therapeutic anticoagulation (Table 2).

While there were decreased thromboembolic events (9\% vs 17.6\% $p=0.476$ ) for patients who received therapeutic anticoagulation, no statistically significant differences were observed. The most common type of thrombotic event was pulmonary embolism (PE) in the thromboprophylaxis group and deep vein thrombosis (DVT) in the therapeutic anticoagulation group (supplementary material). Of note, two of the three new thromboembolic events in the therapeutic dose group occurred after therapeutic anticoagulation was discontinued while patients were transitioned to prophylaxis dosing. 
The therapeutic dose group had a higher rate of major bleeding than the prophylaxis dose group ( $48.5 \%$ vs $14.7 \%, p=0.043$ ). The most common type of major bleeding was grade 3 bleeding requiring red blood cell transfusion over routine transfusion needs (supplementary material).

\section{Discussion}

At a regional teaching hospital in Maryland, we report a beneficial effect of therapeutic anticoagulation in reducing 14day mortality in mechanically ventilated patients with COVID19. To our knowledge, this is one of only a few studies to evaluate therapeutic versus prophylaxis dose anticoagulation in mechanically ventilated COVID-19 patients in the ICU. Parapanjpe et al. analyzed the effect of anticoagulation on mortality in 2,773 COVID-19 patients and reported that longer duration of therapeutic anticoagulation was associated with a reduced risk of mortality ${ }^{14}$. Recently, Trinh et al. demonstrated a marked reduction in mortality in 161 mechanically ventilated patients with COVID-19 who received therapeutic anticoagulation, suggesting that early initiation of anticoagulation after mechanical ventilation can lead to increased survival ${ }^{15}$. Our findings are consistent with the aforementioned studies, with a primary endpoint targeted at 14-day mortality. Profound mortality reduction was observed in therapeutically anticoagulated patients despite having a higher severity of disease than patients in the prophylaxis anticoagulation group. An increased length of stay and duration of mechanical ventilation in therapeutic anticoagulation group can be attributed to increased survival.

These results add to the proposed hypothesis that while mortality in COVID-19 is largely due to hypoxemia secondary to ARDS, an underlying hypercoagulable state marked by high Ddimers may be a contributing factor. Microvascular clot formation induced by vascular and endothelial injury as well as macrovascular thrombosis can result in thromboembolic sequelae leading to worsened patient outcomes ${ }^{16}$. Grasselli et al. discussed a subgroup of COVID-19 patients with ARDS characterized by low static respiratory compliance and high Ddimer concentrations who had markedly increased mortality compared to non-COVID-19 ARDS patients, suggesting additional pathophysiology leading to differences in survival ${ }^{17}$. Although D-dimer values are elevated in the majority of critically ill patients, certain thresholds for screening evaluation may be indicated. Cui et al. reported that venous thromboembolism (VTE) in patients with severe COVID-19 may be related to poor prognosis and significant increases in Ddimer in COVID-19 patients is a good index for identifying highrisk groups for VTE with a high positive predictive value (PPV) ${ }^{18}$. Our institutional ICU algorithm used a D-dimer level greater than $\mathbf{3 0 0 0}$ nanograms per milliliter or an elevation of at least six times baseline as a threshold to recommend therapeutic anticoagulation. However, new thromboembolism event rates in this study were lower than reported in literature for patients receiving thromboprophylaxis, likely attributed to computed tomography (CT) angiography not available for COVID-19 patients and venous ultrasound not performed unless clinically warranted $^{5,19,20}$. Therefore, microvascular and macrovascular thrombosis may remain undetected as recently reported by Nadkarni et $a^{21}$.

Higher rates of major bleeding in therapeutic anticoagulation group indicate an ongoing need to review bleeding risk in individual patients and shared decision making after evaluation of benefits and risk of therapy. Of note, a decrease in 14 and 30-day mortality in therapeutic anticoagulation group was seen despite increased bleeding.

This study is not without limitations which include a retrospective single-center design and small sample size making it less generalizable to hospitals with dissimilar populations. Second, differences in availability of adequate clinician staffing and hospital resources such as personal protective equipment (PPE) and medications (including heparin and its derivatives) need to be addressed when determining the generalizability of this data to other institutions. Patients in the therapeutic anticoagulation group had a higher incidence of receiving corticosteroids, which have shown to reduce mortality in COVID-19 patients, making the results less likely due to the treatment groups alone (supplementary material). This could confound overall results. Suspected pulmonary embolisms were not confirmed by radiography due to CT angiography being difficult to obtain in COVID-19 positive patients. Prophylaxis group and therapeutic anticoagulation had a crossover rate of $38 \%$ and $27.3 \%$ respectively, which can confound overall results. Since our hospital protocol excluded patients with high bleeding risk per HAS-BLED score $>3$ or provider preference, potential benefit of therapeutic anticoagulation cannot be extrapolated to that population. Lastly, our decision to exclude patients who were on mechanical ventilation for less than 72 hours introduces immortal time bias. However, we believe those patients did not receive an appropriate duration or consistency of anticoagulation with achievement of goal therapeutic ranges to be definitely considered in either group.

\section{Conclusion}

In conclusion, therapeutic dose anticoagulation was associated with decreased 14-day and 30-day mortality in mechanically ventilated ICU patients with COVID-19. Due to the increase in major bleeding, risks versus benefits of therapeutic anticoagulation should be weighed in individual cases to assist in clinical decision making. Prospective, multicenter, randomized-controlled trials are needed to determine whether systemic anticoagulation results in a survival benefit in mechanically ventilated patients with COVID-19. 
Acknowledgments: The authors would like to acknowledge Drs. Barry Meisenberg and George Samaras for encouraging research at our institution, in addition to the Luminis Health COVID Clinical Management Committee for incredible collaboration in the midst of the COVID-19 pandemic. In addition, the authors acknowledge Jamie Caldwell and Doug Hosie for providing pharmacy administrative support. Lastly, the authors thank Robert Freeman, Ph.D. for reviewing the statistical analysis within this study.

Conflicts of Interest: The authors have declared no potential conflicts of interest.

Treatment of Human Subjects: IRB review/approval required and obtained

\section{References}

1. Sharma A, Tiwari S, Deb MK, Marty JL. Severe acute respiratory syndrome coronavirus-2 (SARS-CoV-2): a global pandemic and treatment strategies. Int J Antimicrob Agents. 2020; 56 (2): 106054. doi:10.1016/j.ijantimicag.2020.106054

2. Wichmann D, Sperhake JP, Lütgehetmann M, et al. Autopsy Findings and Venous Thromboembolism in Patients With COVID-19: A Prospective Cohort Study. Ann Intern Med. 2020; 173 (4): 268-277. doi:10.7326/M20-2003

3. Yao, Y., Cao, J., Wang, Q. et al. D-dimer as a biomarker for disease severity and mortality in COVID-19 patients: a case control study. J Intensive Care. 2020; 8:49. doi.org/10.1186/s40560-020-00466-z

4. Carsana L, Sonzogni A, Nasr A, et al. Pulmonary postmortem findings in a series of COVID-19 cases from northern Italy: a two-centre descriptive study [published online ahead of print, 2020 Jun 8]. Lancet Infect Dis. 2020; S1473-3099 (20) 30434-5. doi:10.1016/S14733099(20)30434-5

5. Klok FA, Kruip MJHA, van der Meer NJM, et al. Incidence of thrombotic complications in critically ill ICU patients with COVID-19. Thromb Res. 2020; 191: 145-147. doi:10.1016/j.thromres.2020.04.013

6. Auld SC, Caridi-Scheible M, Blum JM, et al. ICU and Ventilator Mortality Among Critically III Adults With Coronavirus Disease 2019. Crit Care Med. 2020; 48 (9): e799-e804. doi:10.1097/CCM.0000000000004457

7. Richardson S, Hirsch JS, Narasimhan M, et al. Presenting Characteristics, Comorbidities, and Outcomes Among 5700 Patients Hospitalized With COVID-19 in the New York City Area. JAMA. 2020; 323 (20): 2052-2059. doi:10.1001/jama.2020.6775

8. McBane RD 2nd, Torres Roldan VD, Niven AS, et al. Anticoagulation in COVID-19: A Systematic Review, Metaanalysis, and Rapid Guidance From Mayo Clinic. Mayo Clin Proc. 2020 Nov;95(11):2467-2486. doi: 10.1016/j.mayocp.2020.08.030.

9. Clinical management of severe acute respiratory infection (SARI) when COVID-19 disease is suspected. WHO/2019nCoV/clinical/2020. 4. World Health Organization; 2020.

10. Thachil J, Tang N, Gando S, et al. ISTH interim guidance on recognition and management of coagulopathy in COVID-19. J Thromb Haemost. 2020; 18 (5): 1023-1026.

doi.org/10.1111/jth.14810
11. COVID-19 Treatment Guidelines Panel. Coronavirus Disease 2019 (COVID-19) Treatment Guidelines. National Institutes of Health; Accessed August 1, 2020.

https://www.covid19treatmentguidelines.nih.gov/

12. Voicu S, Bonnin P, Stépanian A, et al. High Prevalence of Deep Vein Thrombosis in Mechanically Ventilated COVID-19 Patients. J Am Coll Cardiol. 2020; 76 (4): 480-482. doi:10.1016/j.jacc.2020.05.053

13. Trigonis RA, Holt DB, Yuan R, et al. Incidence of Venous Thromboembolism in Critically III Coronavirus Disease 2019 Patients Receiving Prophylactic Anticoagulation. Crit Care Med. 2020; 48 (9): e805-e808. doi:10.1097/CCM.0000000000004472

14. Paranjpe I, Fuster V, Lala A, et al. Association of Treatment Dose Anticoagulation With In-Hospital Survival Among Hospitalized Patients With COVID-19. J Am Coll Cardiol. 2020; 76 (1): 122-124. doi:10.1016/j.jacc.2020.05.001

15. Trinh $M$, Chang $D$, Govindarajulu $U$, et al. Therapeutic Anticoagulation Is Associated with Decreased Mortality in Mechanically Ventilated COVID-19 Patients. medRxiv. 2020.05.30.20117929. doi.org/10.1101/2020.05.30.20117929

16. Iba T, Levy JH, Levi M, Connors JM, Thachil J. Coagulopathy of Coronavirus Disease 2019. Crit Care Med. 2020; 48 (9): 1358-1364. doi:10.1097/CCM.0000000000004458

17. Grasselli G, Tonetti T, Protti A, et al. Pathophysiology of COVID-19-associated acute respiratory distress syndrome: a multicentre prospective observational study [published online ahead of print, 2020 Aug 27]. Lancet Respir Med. 2020; S2213-2600 (20) 30370-2. doi:10.1016/S22132600(20)30370-2

18. Cui S, Chen S, Li X, Liu S, Wang F. Prevalence of venous thromboembolism in patients with severe novel coronavirus pneumonia. J Thromb Haemost. 2020; 18 (6): 1421-1424. doi:10.1111/jth.14830

19. Taccone FS, Gevenois PA, Peluso L, et al. Higher Intensity Thromboprophylaxis Regimens and Pulmonary Embolism in Critically III Coronavirus Disease 2019 Patients [published online ahead of print, 2020 Aug 7]. Crit Care Med. 2020; 10.1097. doi:10.1097/CCM.0000000000004548

20. Voicu S, Bonnin P, Stépanian A, et al. High Prevalence of Deep Vein Thrombosis in Mechanically Ventilated COVID-19 Patients. J Am Coll Cardiol. 2020; 76 (4): 480-482. doi:10.1016/j.jacc.2020.05.053

21. Nadkarni GN, Lala A, Bagiella E, et al. Anticoagulation, Mortality, Bleeding and Pathology Among Patients Hospitalized with COVID-19: A Single Health System Study [published online ahead of print, 2020 Aug 24]. J Am Coll Cardiol. 2020; S0735-1097 (20) 36408-1. doi:10.1016/j.jacc.2020.08.041

22. Wiersinga WJ, Rhodes A, Cheng AC, Peacock SJ, Prescott HC. Pathophysiology, Transmission, Diagnosis, and Treatment of Coronavirus Disease 2019 (COVID-19): A Review [published online ahead of print, 2020 Jul 10]. JAMA. 2020; 10.1001/jama.2020.12839. doi:10.1001/jama.2020.12839. 
Table 1. Baseline patient demographics and clinical scores ${ }^{\mathrm{a}}$

\begin{tabular}{|l|c|c|c|}
\hline \multicolumn{1}{|c|}{ Characteristic $^{a}$} & $\begin{array}{c}\text { Prophylactic } \\
\mathbf{n = 3 4}\end{array}$ & $\begin{array}{c}\text { Therapeutic } \\
\mathbf{n = 3 3}\end{array}$ & P-value \\
\hline Age, years & $66(55.3-73.3)$ & $59.7(50.5-68)$ & 0.144 \\
\hline Male sex, $\mathrm{n}(\%)$ & $17(50 \%)$ & $19(57.6 \%)$ & 0.627 \\
\hline BMI, kg/m & $32.5(26.2-37.3)$ & $30(24.1-36.9)$ & 0.195 \\
\hline Charlson Comorbidity Index & $4(3-5)$ & $3(1.5-3.5)$ & 0.11 \\
\hline APACHE II Score & $24(19-28.3)$ & $23(18-28)$ & 0.496 \\
\hline HAS-BLED Score & $2.5(1-3)$ & $1(0-2)$ & 0.002 \\
\hline CRP, mg/L & $11.9(6.76-17.9)$ & $18.4(11.9-19)$ & 0.014 \\
\hline D-dimer, mcg/mL & $2.55(1.28-3.65)$ & $5.47(3.42-20)$ & $<0.001$ \\
\hline Fibrinogen, mg/dL & $615(519-745)$ & $786.3(620-973)$ & 0.022 \\
\hline Ferritin, ng/mL & $742(370-1357)$ & $1199(623-2406)$ & 0.41 \\
\hline PaO2/FiO2 ratio & $118(71-154)$ & $77(62.5-125.5)$ & 0.09 \\
\hline
\end{tabular}

Abbreviations: BMI, Body Mass Index; CRP, C-Reactive Protein.

${ }^{a}$ Data are presented as median (IQR), unless specified otherwise.

Table 2. Primary, secondary, and other pre-defined endpoints ${ }^{a}$

\begin{tabular}{|c|c|c|c|c|}
\hline \multicolumn{2}{|r|}{ Endpoint } & $\begin{array}{l}\text { Prophylactic } \\
\quad(n=34)\end{array}$ & $\begin{array}{l}\text { Therapeutic } \\
\quad(n=33)\end{array}$ & P-value \\
\hline Primary & 14-day mortality & $14(41.2)$ & $3(9.1)$ & 0.004 \\
\hline \multirow[t]{4}{*}{ Secondary } & 30-day mortality & $18(52.9)$ & $8(24.2)$ & 0.024 \\
\hline & $\begin{array}{l}\text { Duration of mechanical ventilation, days, } \\
\text { mean }( \pm S D)\end{array}$ & $13.3( \pm 11.9)$ & $33.9( \pm 20.2)$ & $<0.001$ \\
\hline & New thromboembolism, events (\%) & $6(17.6)$ & $3(9.1)$ & 0.476 \\
\hline & Major bleed, events (\%) & $7(20.6)$ & $22(66.7)$ & 0.043 \\
\hline Pre-specified & Hospital LOS, mean ( \pm SD) & $26( \pm 17.2)$ & $45.7( \pm 19.2)$ & 0.003 \\
\hline
\end{tabular}

${ }^{\text {aData }}$ are presented number (percent) of patients, unless specified otherwise 
Figure 1. Institutional anticoagulation algorithm to guide clinical decision making regarding thromboprophylaxis or therapeutic anticoagulation in the ICU.

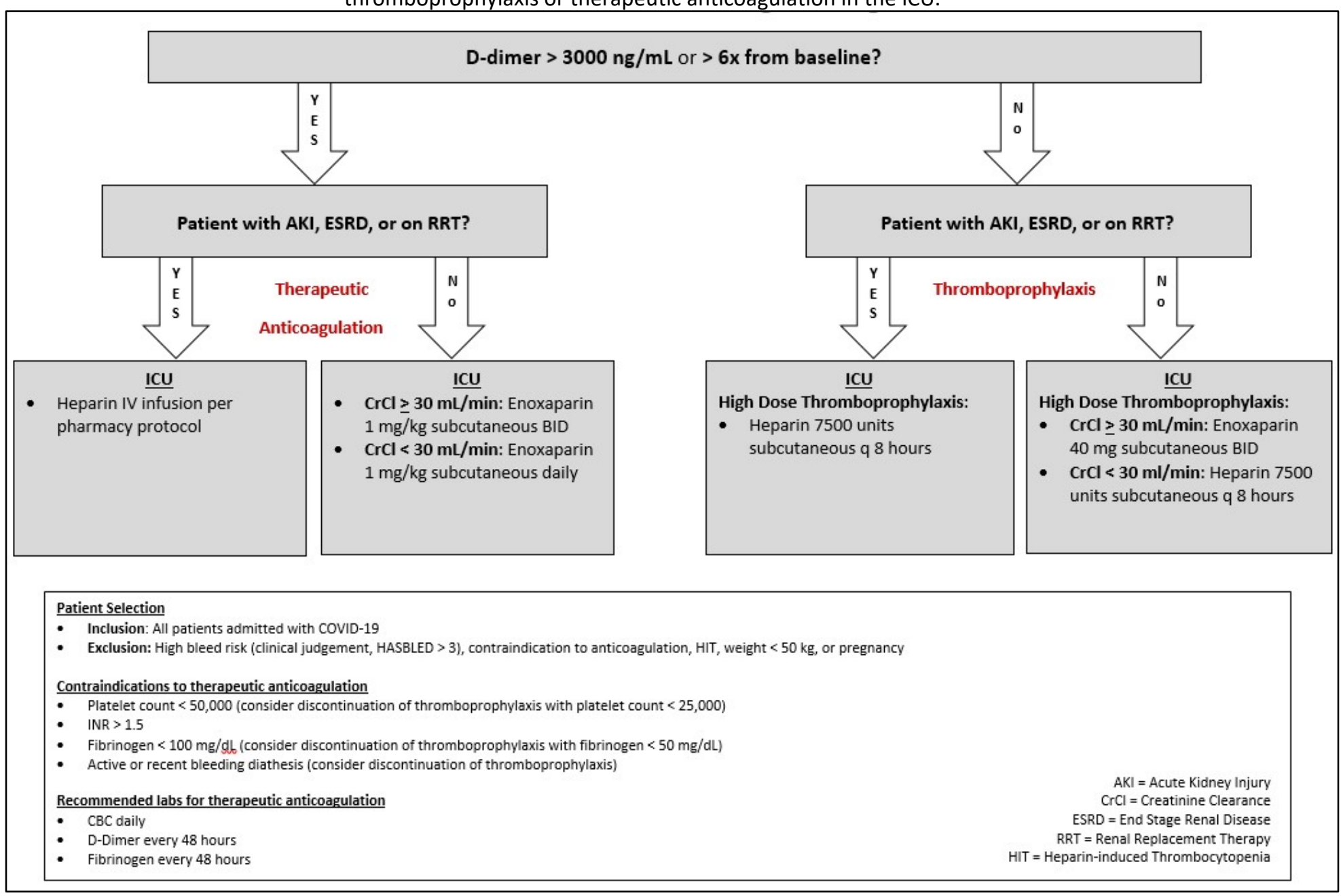

\section{References}

- American Society of Hematology. COVID-19 and coagulopathy: frequently asked questions. From the ASH website. Accessed 2020 Apr 18. Available from https://www.hematology.org/covid19/covid-19-and-coagulopathy

- $\quad$ Chen J et al. Findings of Acute Pulmonary Embolism in COVID-19 Patients. Lancet 2020.

- Cui S, Chen S, Li X, Liu S, Wang F. Prevalence of venous thromboembolism in patients with severe novel coronavirus pneumonia. J Thromb Haemost. 2020

- Deng Y, Liu W, Liu K. Clinical characteristics of fatal and recovered cases of coronavirus disease 2019 (COVID-19) in Wuhan, China: a retrospective study. Chin Med J (Engl). 2020

- Dolhnikoff M et al. Pathological Evidence of Pulmonary Thrombotic Phenomena in Severe COVID-19. Journal of Thrombosis and Haemostasis 2020.

- Fox SE et al. Pulmonary and Cardiac Pathology in COVID-19: The First Autopsy Series from New Orleans. Chemrxiv Pre-Print 2020.

- International Society of Thrombosis and Haemostasis Interim Guidance on Recognition and Management of Coagulopathy in COVID-19. From the ISTH website. Accessed 2020 Apr 18. Available from https://onlinelibrary.wiley.com/doi/epdf/10.1111/jth.14810

- $\quad$ Klok FA et al. Incidence of Thrombotic Complications in Critically III ICU Patients with COVID-19. Thrombosis Research.

- $\quad$ Li T, Lu H, Zhang W. Clinical observation and management of COVID-19 patients. Emerg Microbes Infect. 2020; 9: 687-690.

- Paranjpe I, Fuster V, Lala A, et al. Association of Treatment Dose Anticoagulation with In-Hospital Survival Among Hospitalized Patients with COVID-19. J Am Coll Cardiol. 2020;

- Tang N et al. Anticoagulant Treatment is Associated with Decreased Mortality in Severe Coronavirus Disease 2019 Patients with Coagulopathy. J Thromb Haemost 2020.

- Tang N, Li D, Wang X. Abnormal coagulation parameters are associated with poor prognosis in patients with novel coronavirus pneumonia. J Thromb Haemost. 2020; 18: 844-847.

- $\quad$ Thachil J et al. ISTH Interim Guidance on Recognition and Management of Coagulopathy in COVID-19. Journal of Thrombosis and Haemostasis 2020.

- Thrombosis UK. Practical guidance for the prevention of thrombosis and management of coagulopathy and disseminated intravascular coagulation of patients infected with COVID-19. From the Thrombosis UK website. Accessed 2020 Apr 18. Available from https://thrombosisuk.org/downloads/T\&H\%20and\%20COVID.pdf

- Zhang Y et al. Coagulopathy and Antiphospholipid Antibodies in Patients with COVID-19. NEJM 2020. 
Figure 2. Selection flowchart of study patients.

\section{Evaluated}

121 COVID-19 mechanically ventilated ICU patients who received heparin/enoxaparin

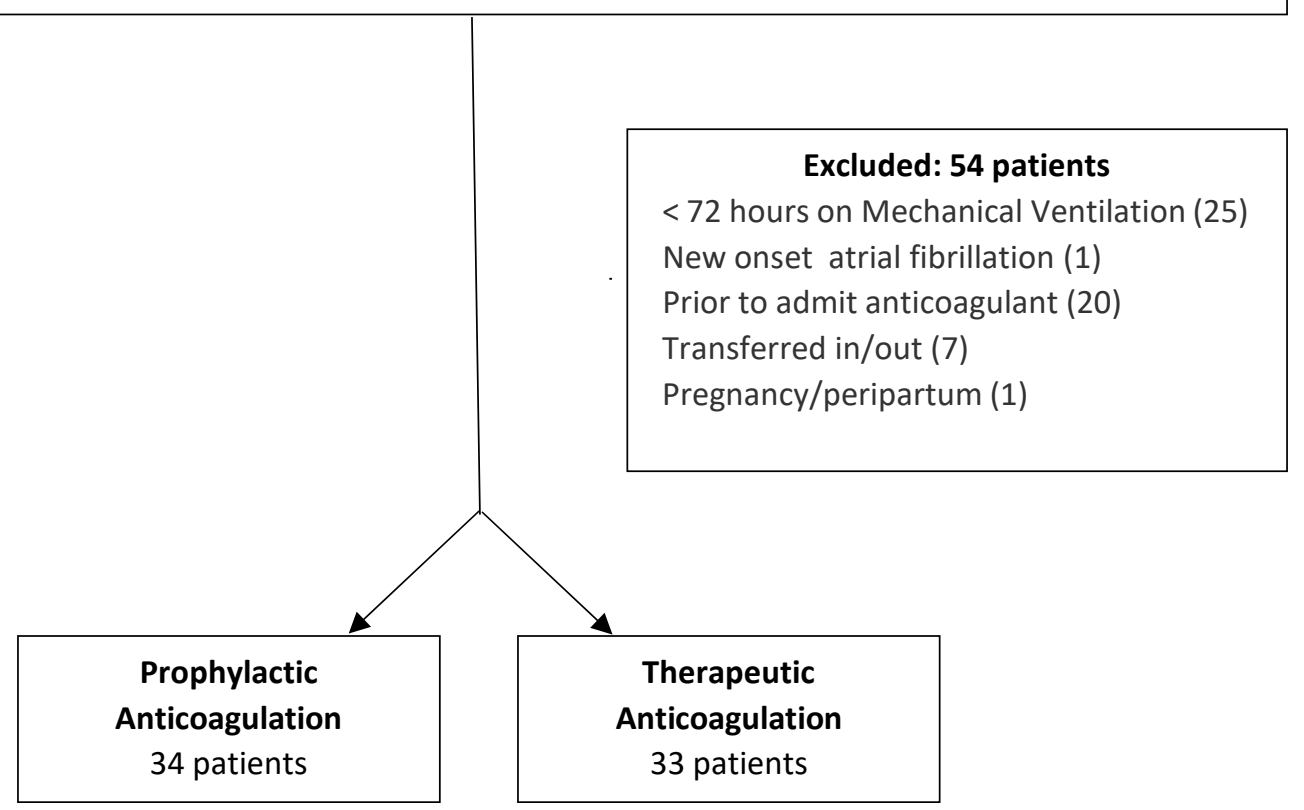

